The Astrophysical Journal, 323:L7-L11, 1987 December 1

1987. The American Astronomical Society. All rights reserved. Printed in U.S.A.

\title{
ON THE ORIGIN OF POSSIBLE DEVIATION FROM THE BLACKBODY SPECTRUM OF THE COSMIC MICROWAVE BACKGROUND RADIATION: INVERSE COMPTON HYPOTHESIS BASED UPON THE EXPLOSION SCENARIO
}

\author{
SATOSHI Yoshioka AND SATORU IKeUCHI \\ Tokyo Astronomical Observatory, University of Tokyo \\ Received 1987 July 6; accepted 1987 September 1
}

\begin{abstract}
Possible deviation from the blackbody spectrum of the cosmic microwave background radiation at the Wien part is indicated by the rocket observation of Nagoya-Berkeley group. Here, the inverse Compton hypothesis is examined based upon the explosion scenario for galaxy formation. Allowed ranges of physical parameters for reproducing the observation are explored in relation to the isotropy of spectrum at the Rayleigh-Jeans part, the size of voids, and the X-ray background radiation.
\end{abstract}

Subject headings: cosmic background radiation — cosmology — galaxies: intergalactic medium

\section{INTRODUCTION}

The discovery of the cosmic microwave background radiation (MBR) in 1964 brought a brilliant victory to the big bang hypothesis over the steady state cosmology. After that, various observations of MBR have presented important constraints on galaxy formation theory. In particular, the Rayleigh-Jeans part at the wavelengths of $10 \mathrm{~cm}$ to $3 \mathrm{~mm}$ indicates the blackbody temperature to be $T_{\mathrm{RJ}}=2.756 \pm$ $0.016 \mathrm{~K}$ (Johnson and Wilkinson 1987), and its surprising isotropy as $|\Delta T / T|<2.1 \times 10^{-5}$ (Uson and Wilkinson 1984) places a severe constraint on the amplitude of density perturbations at the recombination epoch, if the MBR had not suffered scatterings after that.

On the other hand, it was very difficult to measure the temperature and fluctuation of the Wien part of MBR at 1 $\mathrm{mm}$ to $0.4 \mathrm{~mm}$ because of the atmospheric emission. Recently, the Nagoya-Berkeley group have succeeded in measuring the intensity of MBR at the Wien part and presented a beautiful result (Matsumoto et al. 1987). The equivalent blackbody temperature which they have estimated from their observed intensities are as follows:

$$
\begin{aligned}
& \lambda=1.160 \mathrm{~mm} \quad T=2.795 \pm 0.018 \mathrm{~K} \text {, } \\
& 0.709 \mathrm{~mm} \quad 2.963 \pm 0.017 \mathrm{~K} \text {, } \\
& 0.481 \mathrm{~mm} \quad 3.150 \pm 0.026 \mathrm{~K} \text {. }
\end{aligned}
$$

The deviation from the blackbody spectrum is highly probable because their estimated temperature definitely increases with decreasing the wavelength even if the error is included.

What is the origin of this deviation? Two completely different mechanisms are supposed. One is the continuum emission from the dust with the temperature $T_{d} \approx 3 \mathrm{~K}$. The other is the inverse Compton scattering from the Rayleigh-Jeans part of the MBR by hot electrons. For both models, some energy sources are necessary, which heat dusts in the former model and electrons in the latter model. Total necessitated energies are same for both cases, and it is in the order of $\varepsilon_{p}(0) \approx n_{\nu} \times$ $k \Delta T \approx 10^{-14} \mathrm{ergs} \mathrm{cm}^{-3}$ where $n_{\nu}, k$ and $\Delta T$ are the present number density of MBR photons, the Boltzmann constant and the deviation from the unique blackbody temperature. This seems enormously high. For comparison, the rest mass energy of baryons is $\varepsilon_{b}(0) \approx n_{0} m_{p} c^{2} \approx 3 \times 10^{-8} \Omega_{b, 0} h_{100}^{2}$ ergs $\mathrm{cm}^{-3}$. Here, $n_{0}$ is the present number density of baryons, $m_{p}$ is the proton mass, $c$ is the light velocity, $\Omega_{b, 0}$ is the density parameter of baryons, and $h_{100}$ is the Hubble constant in units of $100 \mathrm{~km} \mathrm{~s}^{-1} \mathrm{Mpc}^{-1}$. The energy density of photons changes as $\varepsilon_{p}(z)=\varepsilon_{p}(0)(1+z)^{4}$ and that of baryons as $\varepsilon_{b}(z)=\varepsilon_{b}(0)(1+z)^{3}$. Then, their ratio varies

$$
\varepsilon_{p}(z) / \varepsilon_{b}(z) \approx 3 \times 10^{-7}(1+z) \Omega_{b, 0}^{-1} h_{100}^{-2} .
$$

Since the efficiency of energy ejection by nuclear reactions is at most in the order of $10^{-3}$ (Carr et al. 1984), the following condition must be satisfied

$$
\Omega_{b, 0} h_{100}^{2} /(1+z) \geq 3 \times 10^{-4},
$$

if it is the nuclear energy.

The explosion scenario for galaxy formation by Ostriker and Cowie (1981) and Ikeuchi (1981) presumes the energy injection by exploding stars or quasars, which are assumed to be Population III objects. According to this scenario, the condition (3) is not absurd. In this Letter, we examine the inverse Compton hypothesis for the origin of probable deviation from the blackbody spectrum of MBR based upon the explosion hypothesis.

\section{DETERMINATION OF Y-PARAMETER}

Inverse Compton scattering process with nonrelativistic electrons is described by the Kompaneets equation. Its solution can be determined only by one parameter, the Compton 
$y$-parameter, which is defined as

$$
y=\int_{0}^{\tau}\left(k T_{e} / m_{e} c^{2}\right) d \tau_{\mathrm{T}},
$$

where $T_{e}$ is the electron temperature and $m_{e}$ is the electron mass. The increment of opacity of the Thomson scattering is

$$
d \tau_{\mathrm{T}}=\sigma_{\mathrm{T}} n_{e} d l
$$

where $\sigma_{\mathrm{T}}$ is the Thomson cross section and $n_{e}$ is the number density of electrons. This Kompaneets equation is valid when $h \nu \ll m_{e} c^{2}$ and $k T_{e} \ll m_{e} c^{2}$. The former is safely satisfied for any case, but the latter is dangerous in the present model. We briefly discuss the effect of special relativity in $\S \mathrm{V}$.

When initial photon spectrum is Planckian and $T_{e} \gg T_{r}$ (temperature of radiation), which is easily satisfied in the present case, the Kompaneets equation has a following analytic solution in the Rayleigh-Jeans part of MBR (Zeldovich and Sunyaev 1969):

$$
T_{0}=T_{1} e^{-2 y},
$$

where $T_{0}$ and $T_{1}$ are, respectively, the effective blackbody temperature at the Rayleigh-Jeans part before and after the inverse Compton scattering. We take the observed RayleighJeans part $T_{1}=T_{\mathrm{RJ}}=2.70 \mathrm{~K}$. Using the blackbody spectrum with $T_{0}$, the spectrum at the Wien part is compared with the Planck spectrum for a fixed $y$ and we finally obtain the effective blackbody temperature $T_{B}(\nu ; y)$. Comparing $T_{B}(\nu ; y)$ with equation (1) we determine the best value for $y$ as $y=0.02$.

\section{EXPLOSION SCENARIO}

Basing their work upon the explosion scenario for galaxy formation, Yoshioka and Ikeuchi (1987) have examined the formation process of large voids. At the same time they have studied thermal process in association with explosions. Here, we utilize their results without going to details.

\section{a) Models}

For the density parameters we tentatively assume the following baryon-dominated universe: (1) $\Omega_{0}=\Omega_{b, 0}=1.0$, (2) $\Omega_{0}=\Omega_{b, 0}=0.1$, where $\Omega_{0}$ and $\Omega_{b, 0}$ are the present density parameter of the universe and that of baryons. We suppose that the seed objects with mass $M_{i}$ explosively release energy $E_{i}$ at the redshift $z_{i}$ with the efficiency $\varepsilon$. Then we have (Carr, Bond, and Arnett 1984)

$$
E_{i}=1.8 \times 10^{61}\left(\varepsilon / 10^{-4}\right)\left(M_{i} / 10^{11} M_{\odot}\right) \text { ergs } .
$$

The shock waves generated by explosions propagate in the universe and heat up the general intergalactic gas (IGG). Electrons heated up to $T_{e}=10^{\sim-9} \mathrm{~K}$ transform the photons at the Rayleigh-Jeans part to the Wien part by inverse Compton scatterings.

We assume the initial space density of seeds to be $N_{s, i}$, which is conserved in the comoving coordinate $N_{s}(z)=$ $N_{s, i}(1+z)^{3} /\left(1+z_{i}\right)^{3}$, and the expanding shock waves would overlap at $z=z_{\text {over }}$. A simple overlapping condition

$$
\frac{4 \pi}{3} R_{s}^{3}\left(z_{\text {over }}\right) N_{s}\left(z_{\text {over }}\right)=1
$$

gives the initial seed number

$$
N_{s, i}=\frac{3}{4 \pi}\left[R_{s}\left(z_{\text {over }}\right)\left(\frac{1+z_{i}}{1+z_{\text {over }}}\right)\right]^{-3}
$$

where $R_{s}\left(z_{\text {over }}\right)$ is the shell radius at $z_{\text {over }}$. Then, independent parameters, which characterize the present model, are $E_{i}, z_{i}$, and $z_{\text {over }}$ except for the cosmological parameter $\Omega_{0}$ and $\Omega_{b, 0}$.

\section{b) Basic Equations}

By using the thin shell approximation, the equation of motion of the shell is written as (Ostriker and McKee 1984)

$$
\frac{d}{d t}\left(M_{s} V_{s}\right)=4 \pi R_{s}^{2}\left[P+\rho_{b} V_{\mathrm{H}}\left(V_{s}-V_{\mathrm{H}}\right)\right]-\frac{G M_{s}}{2 R_{s}^{2}},
$$

where $V_{s}$ and $V_{\mathrm{H}}$ are the expanding velocity of the shell and the unperturbed Hubble velocity, $\rho_{b}$ and $P$ are the average gas (baryon) density and pressure inside the shell, and $M_{s}=$ $4 \pi R_{s}^{3} \rho_{b} / 3$ is the mass swept to the shell.

The total energy $E$ of the shell is the sum of kinetic $E_{\text {kin }}=M_{s} V_{s}^{2} / 2$, and thermal $E_{\mathrm{th}}=4 \pi R_{s}^{3}(3 P / 2) / 3$ energy, and it decreases by the energy loss

$$
\frac{d E}{d t}=-\int_{v} \Lambda d V
$$

where $\Lambda$ is the cooling rate per unit volume (Umemura and Ikeuchi 1984) and the integral is taken over the volume of thin shell. The enhanced density behind the shock front is taken as $\rho=D \rho_{b}$, where the enhancing factor $D$ is assumed to be 10 (Ikeuchi, Tomisaka, and Ostriker 1983). The electron temperature at the shell $T_{e}$ is easily calculated $T_{e}=$ $\left(E_{\mathrm{th}} / M_{s}\right)(2 \mu / 3 k), \mu$ being the mean particle mass. The above equations (10) and (11) are integrated for a given set of parameters $\left(E_{i}, z_{i}, z_{\text {over }}\right)$.

The $y$-parameter is defined by

$$
\begin{aligned}
y= & \int_{z_{i}}^{0} \frac{k T_{e}(z) f_{h}(z)}{m_{e} c^{2}} \sigma_{T} n_{e}(z) c\left(\frac{d t}{d z}\right) d z \\
= & 9.98 \times 10^{-12} h_{100} \Omega_{b, 0} \int_{0}^{z_{i}} T_{e}(z) f_{h}(z)(1+z) \\
& \times\left(1+\Omega_{0} z\right)^{-1 / 2} d z .
\end{aligned}
$$

Since the hot gas regions are clumpy the filling factor $f_{h}(z)$ of them is multiplied. It is defined as

$$
\begin{array}{rlrl}
f_{h}(z) & =\left[R_{s}(z)(1+z) / R_{s}\left(z_{\text {over }}\right)\left(1+z_{\text {over }}\right)\right]^{3} \\
& =1 & \text { at } z>z_{\text {over }}, \\
& \text { at } z<z_{\text {over }} .
\end{array}
$$


No. 1,1987

TABLE 1

Calculated Models

\begin{tabular}{|c|c|c|c|c|c|c|}
\hline Model & $\Omega_{0}$ & $\Omega_{b, 0}$ & $z_{\text {over }}$ & Minimum $E_{i}$ & $\varepsilon \Omega_{b, 0}^{B} / 10^{-3}$ & $R_{s}(0)(\mathrm{Mpc})$ \\
\hline A. & 1.0 & 1.0 & 3.0 & $10^{64-65} \mathrm{ergs}$ & $\sim 0.04$ & $>10$ \\
\hline B. & 1.0 & 1.0 & 10.0 & $10^{63-64}$ & $\sim 0.04-0.05$ & $>5$ \\
\hline C. & 0.1 & 0.1 & 3.0 & $10^{66}$ & $\sim 0.04$ & $>20$ \\
\hline D & 0.1 & 0.1 & 10.0 & $10^{65-66}$ & $\sim 0.04$ & $>15$ \\
\hline
\end{tabular}

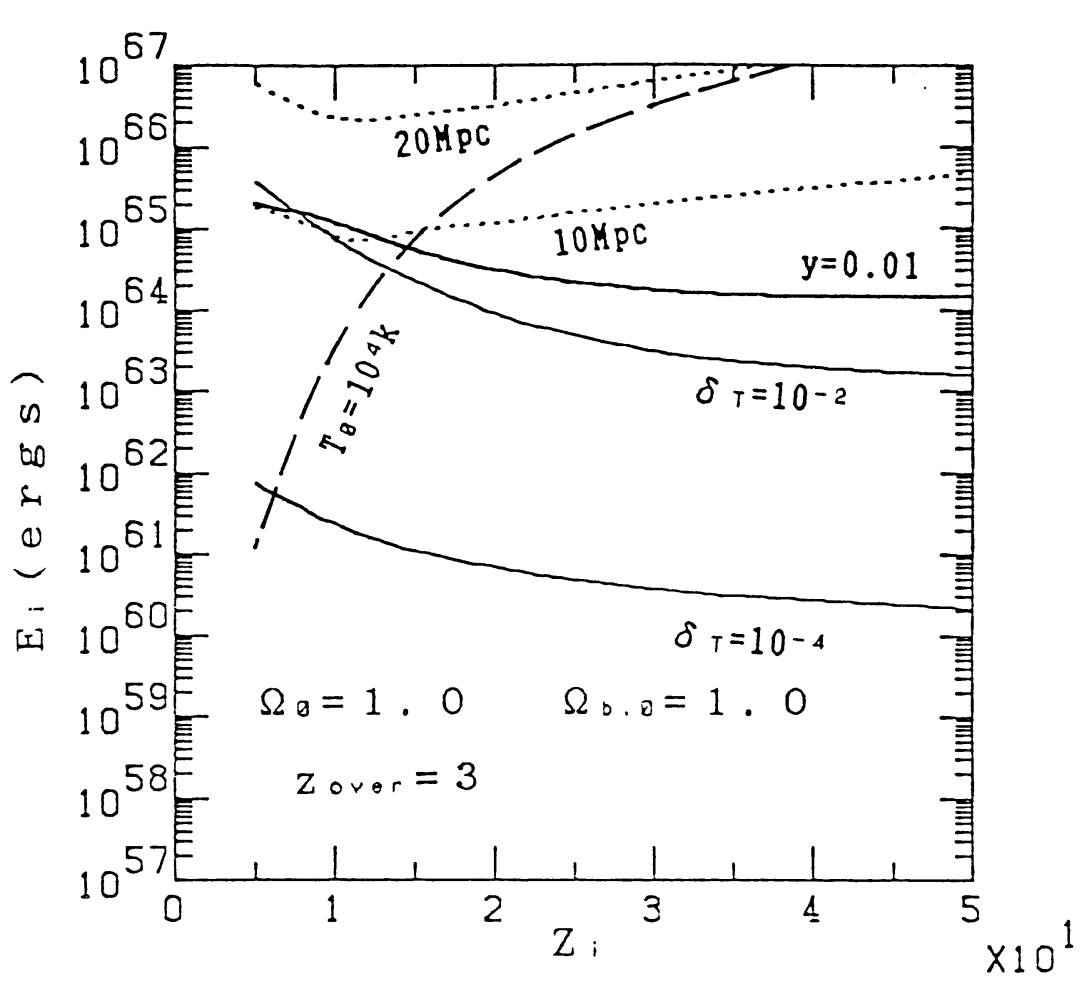

FIG. 1.-Contour maps of $y$ (heavy solid lines), $R_{s}(0)$ (dotted line), $T_{I}(0)$ (dashed line), and $|\Delta T / T|$ (solid line) in the $E_{i}-z_{i}$ plane for case $\mathrm{A}$

\section{c) Results}

In Table 1 , we tabulated parameters $\Omega_{0}, \Omega_{b, 0}$ and $z_{\text {over }}$ of examined models.

In Figure 1, we illustrate in the $E_{i}-z_{i}$ plane the contour maps of the $y$-parameter (heavy solid lines), the present radius of a shell $R_{s}(0)$ (dotted lines), and the present temperature of IGG $T_{I}(0)$ (dashed lines) for case $\mathrm{A}$ in Table 1. After the overlapping, we suppose the shell expands with cosmic expansion, $R_{s}(0)=R_{s}\left(z_{\text {over }}\right)\left(1+z_{\text {over }}\right)$. The necessitated value of $y \approx 0.02$ is obtained almost independent of $z_{i}$ at $E_{i} \approx 10^{64-65}$ ergs. However, if we constrain the size of the shell $R_{s}(0)>10$ Mpc as usual voids (de Lapparent, Geller, and Huchra 1986) it leads to $z_{\mathrm{i}} \leqslant 20$.

For case B with $z_{\text {over }}=10$, the energy injection occurs at $z>10$ and the blackbody spectrum is easily deformed by efficient Compton scatterings. This can be seen from a relatively high value of $y$. On the other hand, as the results of the early overlapping and the efficient Compton cooling the shell can not expand much. With decreasing $z_{\text {over }}$ two competitive effects work comparatively.
In Figure 2, we show the results of case D for comparison. In such a low-density universe, the shell can expand easily so large as $R_{s}(0) \approx 20 \mathrm{Mpc}$, but the Compton scatterings are not so efficient because of low number density of hot electrons. In order to save the latter point it must be $E_{i} \gtrsim 10^{65.5}$ ergs.

In Table 1, we summarize the minimum energy for reproducing condition $y \geq 10^{-2}$ and the present shell radius $R_{s}(0)$. The resultant ejection energy density is obtained as $\varepsilon_{\mathrm{ej}}=$ $E_{i} N_{s, i}$, which is transformed to the condition for the equivalent burnt-out mass at the present epoch using the efficiency of energy release $\varepsilon$,

$$
\varepsilon \Omega_{b, 0}^{B} \geq E_{i} N_{s, i} /\left[c^{2} \rho_{\mathrm{cr}, 0}\left(1+z_{i}\right)^{3}\right]
$$

where $\rho_{\mathrm{cr}, 0}$ is the critical density at the present epoch. For each model, $\varepsilon \Omega_{b, 0}^{B}$ is also tabulated. As is seen, $\varepsilon \Omega_{b, 0}^{B} \approx$ $(0.4-0.5) \times 10^{-4}$. If the energy source is the nuclear energy, $\varepsilon \leqslant 10^{-3}$. Therefore, $\Omega_{b, 0}^{B}$ must be larger than $0.04-0.05$. 


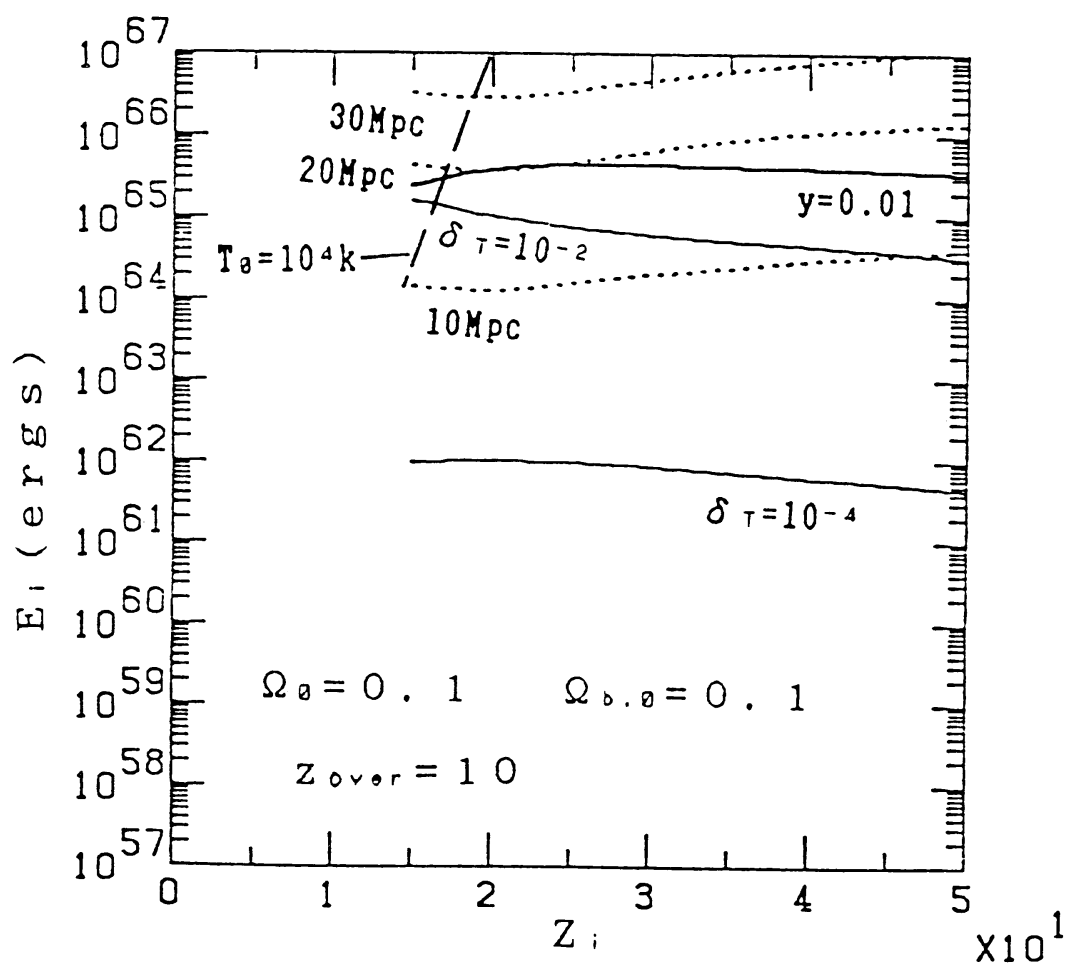

FIG. 2.-Same as Fig. 1 but for case D

For any case, the temperature of IGG becomes much lower than $10^{4} \mathrm{~K}$ for the model of $z_{i}>15$ and is higher than $10^{4} \mathrm{~K}$ for $z_{i}<15$. Then, the Gunn-Peterson test demands the reheating and reionization in the former case.

On the other hand, the contribution of hot gas to the X-ray background radiation at $E_{x}=\sim 2-10 \mathrm{keV}$ should not exceed the observed one (Marshall et al. 1980). This gives the upper limit to the explosion redshift as $z_{i} \gtrsim 10$ for case A.

\section{DISCUSSION}

\section{a) Fluctuation of $M B R$}

In the preceding section we discarded the induced anisotropy of MBR. Generally speaking, the explosions might occur randomly in space, so that the number of explosions contained in the observed beam should fluctuate (Hogan 1984; Vishniac and Ostriker 1986). Yoshioka and Ikeuchi (1987) have carefully examined the anisotropy of MBR due to the random explosions in consideration of relative sizes between the beam width of telescope and the shell size. The cross sections of beam and shell are, respectively, given by $A_{b}=\pi(B / 2)^{2}$ and $A_{s}=\pi R_{s}^{2}(z), B$ being related to the beam width. The interpolation formula for the anisotropy of MBR is

$$
\begin{aligned}
|\Delta T / T|= & \llbracket \int_{z_{i}}^{0}\left\{\left(\frac{2}{3}\right)\left[\frac{\sigma T}{A_{c}(z)}\right]\left[\frac{E_{\mathrm{th}}(z)}{m_{e} c^{2}}\right]\right\}^{2} \\
& \times N_{s}(z) A_{c}(z) c\left(\frac{d t}{d z}\right) d z \rrbracket,
\end{aligned}
$$

where we utilize the effective cross section $A_{c}=\pi[B / 2+$ $\left.R_{s}(z)\right]^{2}$. Since the explosion scenario gives $A_{c}(z), E_{\mathrm{th}}(z)$ and $N_{s}(z)$, we can calculate $|\Delta T / T|$ by using equation (15). We take the angular beam width as $\Delta \theta=1.5$.

In Figures 1 and 2, we also plot the contours $|\Delta T / T|=$ $10^{-2}$ and $10^{-4}$ by solid lines. This indicates that if the deviation from the blackbody spectrum of MBR at the Wien part is originated in the inverse Compton effects of $y \approx 10^{-2}$, they inevitably produce the anisotropy of MBR in the order of $|\Delta T / T| \approx 10^{-2}$. Since the upper limit of the anisotropy at the Rayleigh-Jeans part is so small as $\sim 2 \times 10^{-5}$ (Uson and Wilkinson 1984), the anisotropy of MBR due to the inverse Compton effects should be erased by the Thomson scattering by the electrons in ionized IGG. A rough criterion of this erasing effect will be that the optical depth exceeds about $\ln \left(\sim[2-3] \times 10^{2}\right)$

$$
\tau_{\mathrm{T}}=\int_{z_{i}}^{0} \sigma_{\mathrm{T}} n_{e}(z) c\left(\frac{d t}{d z}\right) d z \geq 5 .
$$

This condition is rewritten as

$$
\begin{aligned}
& \Omega_{b, 0} h_{100}\left(1+z_{i}\right)^{3 / 2} \gtrsim 130 \quad \Omega_{0}=1, \\
& \Omega_{b, 0} h_{100}\left(1+z_{i}\right)^{2} \gtrsim 160 \quad \Omega_{0} \ll 1.0,
\end{aligned}
$$

where we assume $1+z_{i} \gg 1$. This result indicates that the explosions and inverse Compton effects had to occur at $z_{i} \gtrsim 40$ for consistent value of $\Omega_{b, 0}$. 


\section{b) Effects of Special Relativity}

From the condition that the $y$-parameter should be in the order of $10^{-2}$, the initial temperature of IGG might be

$$
T_{i}=3.5 \times 10^{9}\left(y / 10^{-2}\right) h^{-1} \Omega_{b, 0}^{-1}\left(1+z_{i}\right)^{-3 / 2}\left(\Omega_{0}=1\right) .
$$

Therefore, the hot electrons may be considered to be relativistic, $k T_{e} \approx m_{e} c^{2}$. In this case, although we must abandon the Kompaneets equation, which is valid only for $k T_{e} \ll m_{e} c$, we examine only the relativistic effect of scatterings in the present Letter. We calculate the photon spectrum which suffers the Compton scatterings with optically thin relativistic electron gas following Wright (1979).

The relativistic effect considerably increases the increment of effective temperature at the Wien part in the result of scatterings with large energy shifts. The consistent set of parameters $T_{e}, T_{0}$, and $y$ with observations would be

$$
\begin{aligned}
T_{e} & \approx(0.5-1) \times 10^{9} \mathrm{~K}, \quad T_{0} \approx 2.8 \mathrm{~K}, \quad y \approx 0.01-0.02, \\
\tau & \approx 0.1-0.2
\end{aligned}
$$

\section{CONCLUSION}

Summing up the results in $\S$ III and the above, the following time sequence in the explosion scenario would be probable:

$$
\begin{aligned}
& z_{i} \approx 50: \quad \text { explosions of } E_{0} \approx 10^{65} \text { ergs and } \Omega_{b, 0}^{B} \geq 0.04 ; \\
& \text { inverse Compton scattering of MBR; } \\
& z \approx 50-10: \text { smearing out of anisotropy of MBR by the } \\
& \text { Thomson scattering; } \\
& z \approx 10: \quad \text { overlapping of shells; } \\
& z \approx 5: \quad \text { fragmentation of shells and formation of } \\
& z \approx 4:
\end{aligned}
$$

We would like to thank S. Hayakawa and T. Matsumoto for their stimulating discussion.

\section{REFERENCES}

Carr, B. J., Bond, J. R., and Arnett, W. D. 1984, Ap. J., 277, 445 de Lapparent, V., Geller, M., and Huchra, J. 1986, Ap. J. (Letters), 302, L1.

Hogan, C. 1984, Ap. J. (Letters), 284, L1.

Ikeuchi, S. 1981, Pub. Astr. Soc. Japan, 33, 211

Ikeuchi, S., Tomisaka, K., and Ostriker, J. P. 1983, Ap. J., 265, 583

Johnson, D. G., and Wilkinson, D. T. 1987, Ap. J. (Letters), 313, L1.

Marshall, F. E., Boldt, E. A., Holt, S. S., Miller, R. B., Mushotzky, R. F.,

Rose, L. A., Rothschild, R. E., and Serlemitsos, P. J. 1980, Ap. J., 235, 4 .
Matsumoto, T., Hayakawa, S., Matsuo, H., Murakami, H., Sato, S., Lange, A. E., and Richards, P. L. 1987, Ap. J. (Letters), submitted. Ostriker, J. P., and Cowie, L. L. 1981, Ap. J. (Letters), 243, L127. Ostriker, J. P., and McKee, C. F. 1984, preprint.

Umemura, M., and Ikeuchi, S. Progr. Theor. Phys. (Kyoto), 72, 47. Uson, J. M., and Wilkinson, D. T. 1984, Nature, 312, 427. Vishniac, E. T., and Ostriker, J. P. 1986, Soc. Italiana Fis., 1, 157. Wright, E. L. 1979, Ap. J., 232, 348.

Yoshioka, S., and Ikeuchi, S. 1987, Pub. Astr. Soc. Japan, submitted. Zeldovich, Ya. B., and Sunyaev, R. A. 1969, Ap. Space Sci., 4, 301.

SATORU IKEUCHI and SATOSHI YoShioka: Tokyo Astronomical Observatory, University of Tokyo, Mitaka, Tokyo 181, Japan 